\title{
Experimental investigation of main rotor wake
}

\author{
Robert Stepanov ${ }^{1 *}$, and Sergey Mikhailov ${ }^{1}$ \\ ${ }^{1}$ Kazan National Research Technical University named after A.N. Tupolev, Department of \\ Aerohydrodynamics, 420111, 10 Karl Marx Street, Kazan, Russian Federation
}

\begin{abstract}
In this work, experimental results of rotor wake in hover mode are presented. The experiments were carried out with a rotor rig model in the T-1K wind tunnel in Kazan National Research Technical University (Kazan Aviation Institute). The rotor consisted of four identical blades. The Q-criterion was used to identify tip vortices for a $2 \mathrm{D}$ case. The results were then compared with two different wake models.
\end{abstract}

\section{Introduction}

Experimental investigation of velocity fields in the wake of the helicopter rotor is a challenging task. Experimental investigation of real rotor wake is very problematic due to complex nature of the flow field and high sensitivity of the rotor due to its operating conditions. The underlying physics of the rotor wake behaviour is very similar to that of a finite wing, but they have differences due to the proximity and mutual interference of vortices trailed from the rotor blades [1].

Much research has been conducted in the past, focusing on the rotor wake characteristics and tip vortices. The rotor wake behaviour is defined by a flight regime in which it operates. McCroskey [2] and Landgrebe [3] carried out comprehensive reviews on different approaches, used to model helicopter rotor wakes.

Being able to predict the rotor's wake plays an important role during the preliminary structural calculations of the rotor blades and overall aerodynamic characteristics of the helicopter during the design phase. At the same time, the underlying physics of the vortex formation and evolution of the tip vortices, trailed from the helicopter rotor blades, is not fully understood and requires further attention $[1,4]$.

$\mathrm{Up}$ to the date many physical aspects of rotor tip blade vortex formation and evolution as well as of rotor wake's behaviour are not well understood. This work outlines some of the experimental results, which were obtained using PIV. In this work, the wake of rotor model is investigated in hover mode.

\footnotetext{
* Corresponding author: rpstepanov@kai.ru
} 


\section{Vortex identification}

In this work, vortices were identified using Q-criterion, which defines vortices as connected spatial regions, where the Euclidean norm of the vorticity tensor $\boldsymbol{\Omega}$ dominates over the rate of strain tensor $\boldsymbol{S}[5]$ :

$$
Q=0.5\left[|\boldsymbol{\Omega}|^{2}-|\boldsymbol{S}|^{2}\right]>0
$$

Due to the fact that only one camera was used in the experiments, vorticity tensor $\boldsymbol{\Omega}$ and the rate of strain tensor $\boldsymbol{S}$ in equation (1) are reduced to a two-dimensional form:

$$
\begin{gathered}
|\boldsymbol{\Omega}|^{2}=(\partial u / \partial y-\partial v / \partial \mathbf{x})^{2} \\
|\boldsymbol{S}|^{2}=2(\partial u / \partial x)^{2}+2(\partial v / \partial y)^{2}+(\partial u / \partial y+\partial v / \partial x)^{2}
\end{gathered}
$$

Different prescribed wake models exist for obtaining tip vortex positions for hovering flight regimes. Landgrebe's model [6] is based on experimental studies of various rotor models with different number of blades, blade twist and aspect ratio. Later, Kocurek \& Tangler [7] derived a prescribed wake model, which was based on a different set of experimental results. Kocurek \& Tangler's model was similar to Landgrebe's model, but included blade lift distribution for the modelling of the axial settling rates and did not use inner vortex sheets.

\section{Experimental setup}

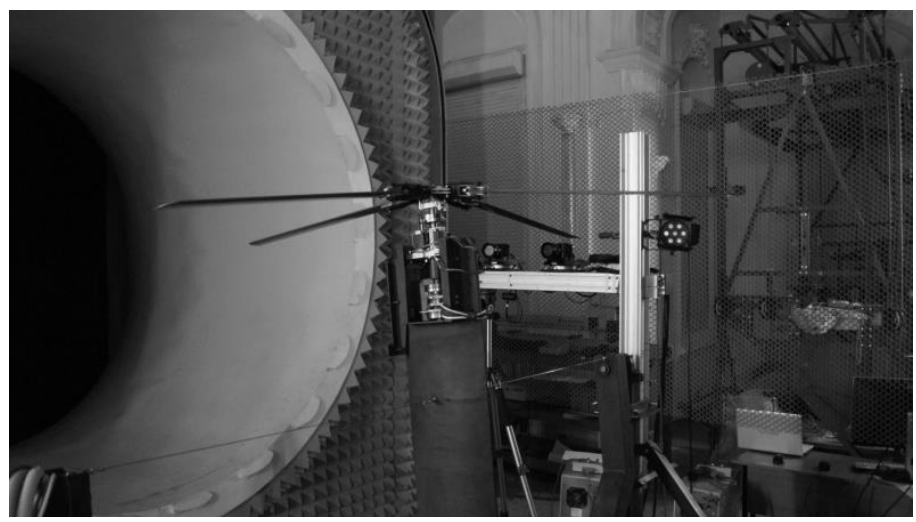

Fig. 1. Experimental setup in the $\mathrm{T}-1 \mathrm{~K}$ wind tunnel.

All results were obtained in $\mathrm{T}-1 \mathrm{~K}$ wind tunnel at the Kazan National Research Technical University (KNRTU-KAI) named after A.N. Tupolev.

The four-bladed rotor rig (see Figure 1) was used to study its wake in hover mode. It was placed at the center of the test section, so that the distance from the rotor plane to ceiling was $2.6 \mathrm{~m}$, and the distance from the rotor plane to the ground was $2.1 \mathrm{~m}$. The direction of the flow is shown in Figure 2 (b).

The rotor had the diameter of $1640 \mathrm{~mm}$, the blades had a constant chord of $65 \mathrm{~mm}$ along their span with rounded tips and no twist. The blades were attached to an articulating hub with three hinges. The collective pitch angle was set at $8^{\circ}$. The rotor's angular speed was fixed at $900 \mathrm{rpm}$. The blade tip Mach number was $\mathrm{M}_{r}=0.23$ and Reynolds number at $75 \%$ of the rotor radius was $R e_{0.75 r}=2.57 \times 10^{5}$. 


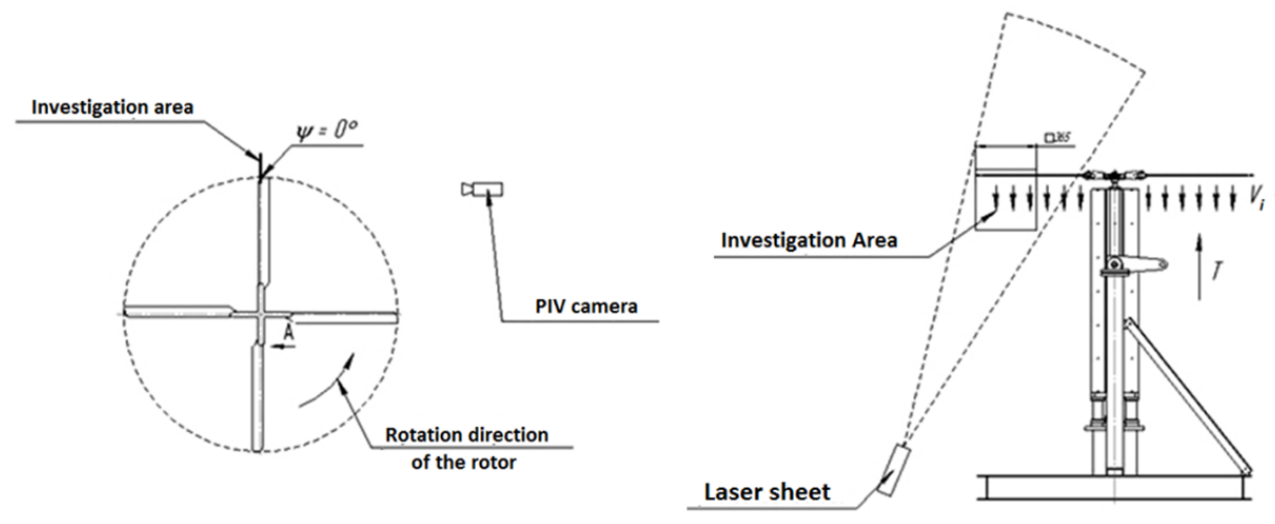

(a) Top view

(b) Side view

Fig. 2. Schematic of the experimental setup: (a) top view; (b) side view.

Velocity fields were obtained with Dantec PIV system, which comprised of $\mathrm{Nd}-\mathrm{YaG}$ laser Litron 425-10 with maximum output of $425 \mathrm{~mJ}$, exposure time of $6 \mathrm{~ns}$ and wave length of $532 \mathrm{~nm}$. Exposure frequency during the experiments was set at $8 \mathrm{~Hz}$. The flow was seeded with olive oil particles using a liquid seeding generator.

The PIV camera FlowSenseEO 4 M, with the resolution of $2048 \times 2048$ pixels, was positioned to capture the tip vortices trailing from the rotor blades, as shown in Figure 2.

\section{Results and discussion}

As it was already mentioned, Q-criterion was used to identify vortex positions in the wake of the rotor in hover mode. The velocity fields, which were obtained using the PIV system, were used to calculate Q-criterion values for the 2D case using equations (1), (2) and (3).

One of the exposure frames is shown in Figure 3 (a). Here, four distinct vortices can be seen, which are shown as shaded colored regions where $Q>0$. In addition, the velocity field and streamlines are shown on the same figure. It is worth noting that the positions of the vortices, obtained using the Q-criterion, do not coincide with the points where the resultant velocity is equal to null, but they are rather located closer to the rotor's axis of rotation.

Multiple velocity fields were obtained during the experiment, each corresponding to a certain exposure frame, taken at a different point of time. The collection of each vortex position for each exposure frame is shown in Figure 3 (b) with dots. Those coordinates were then approximated by a polynomial line. The dots on the plot represent points in the vortices with highest $Q$-values.

Fluctuations of the vortices from their mean values indicate that there is aperiodicity in the wake, also known as vortex wondering. It is also evident, that wake aperiodicity increases with the wake's age.

Comparison of prescribed wake models show that Kocurek \& Tangler's model better agrees with experiments, especially in the early age of the wake. 
(a)

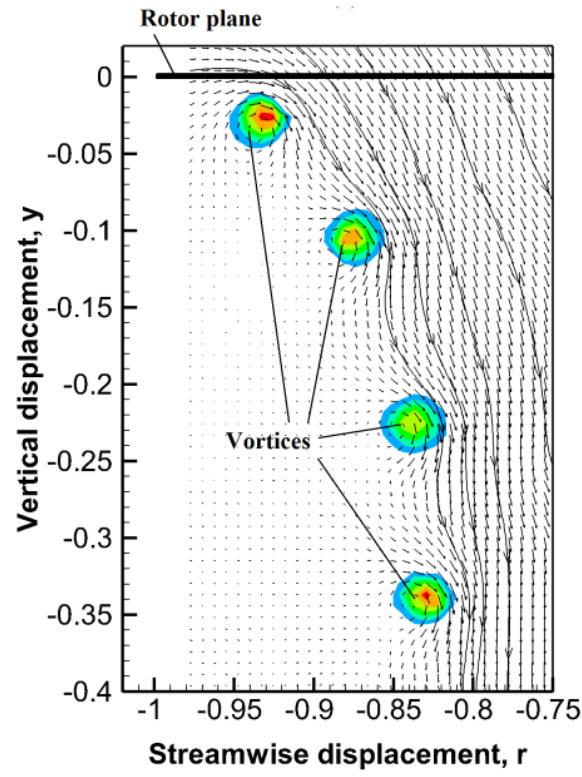

(b)

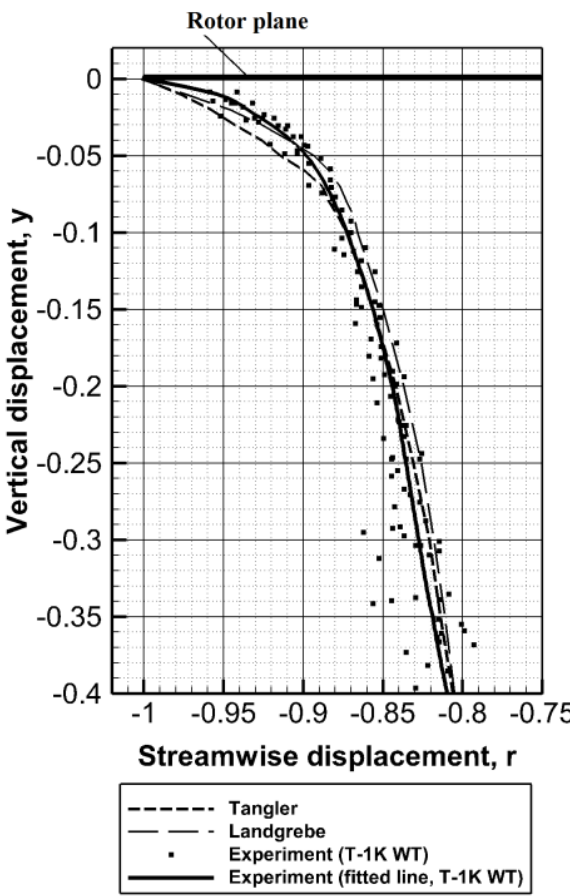

Fig. 3. Experimental results: (a) a typical exposure frame, featuring tip vortices $(Q>0)$; (b) comparison of the experimental data with prescribed wake models.

\section{Conclusions}

This work presents experimental results of wake investigation using PIV system. Tip vortices were identified using Q-criterion for a two-dimensional case. The wake data is then compared with Landgrebe' prescribed wake model and Kocurek \& Tangler's prescribed wake model. The results also reveal that there is aperiodicity inherent to the flow.

This work was supported by the grant 'Numerical and physical modelling of aerodynamic and aeroacoustic characteristics of rotor systems of future concept aircarft' (No. 9.1577.2017/ P Ch) of the Ministry of Education and Science of the Russian Federation.

\section{References}

1. G.J. Leishman, Principles of Helicopter Aerodynamics (Cambridge University Press, Cambridge, U.K., 2006)

2. W.J. McCroskey, 33rd AIAA ASME, 95-0530 (1995)

3. A.J. Landgrebe, 2nd Int. Conference on Rotorcraft Basic Research (1988)

4. J.G. Leishman, A. Bagai, AIAA J. 36, 415 (1998)

5. G. Haller, J. Fluid Mech. 5251 (2005)

6. A. J. Landgrebe, J. American Helicopter Soc. 17, 2 (1972)

7. J.D. Kocurek, J.L. Tangler, J. American Helicopter Soc. 21, 24 (1976) 